\title{
Influence of Large-scale PV Integration on Voltage and Feeder Loss in Distribution Network
}

\author{
Qiang Li1,a,Dan Liu1,b,Xiaodong Yuan¹,c \\ 1State Grid Jiangsu Electric Power CompanyNanjing, P.R. China \\ a35830342@qq.com,b649711458@qq.com,clannyyuan@hotmail.com
}

Keywords:DG;large-scale PV;distribution network; feeder loss; voltage

\begin{abstract}
Influence on the feeder loss and voltage might be brought in with large-scale PV connected. It is important to find out the dynamic change law in thorough quantitative analysis. In this paper, the distribution of voltage on the feeder is calculated. Analytical expressions have been derived to determine the limit value of the power that can be injected into the distribution network and the impacts of voltage passing the limit are studied. An example where different PV integration types (centralized or decentralized) and different grid operation conditions are evaluated and compared. Results show that the integration of PV can help to reduce the loss; the centralized and decentralized integration is fit for different kind of load distributions along the line.
\end{abstract}

\section{Introduction}

Distributed generation (DG) is becoming more and more important in the future generation system. The distribution network with PV integration connected got important benefits in terms of reduction of losses, and reduction of congestion in branches. [1] With the deepening of the research on PV integration, scientific and quantitative research methodology has been used in the analyses of the feeder loss and voltage.

A representative distribution feeder was selected for analyzing the impact of different penetrations of PV on the feeder voltage regulation. Two extreme scenarios, peak load and maximum reverse power flow, were simulated in order to study the feeder's voltage performance under different conditions. [2-4] A new methodology for coordinated voltage support in distribution networks with large integration of distributed generation and micro-grids is given. [5] An optimization tool based on a meta-heuristic approach was developed to address the voltage control problem. [6] Y. Liu's study assesses the effects of a high penetration of distributed PV on thedistribution system voltage control, and on the associated reactive power flow through the distribution system.

\section{Impact of Large-scale PV Integration on Voltage and Line Loss of Distribution Grid}

Fig. 1 shows a typical distribution network with single PV integration. Thedistributionnetwork radiating from the transformer's secondary to the surrounding stretch, supplying power for the load. There are $N$ loads in the distribution network. The $i t h(i=1,2, \ldots, N)$ load's power consumption is $P_{i}+j Q_{i}$, the unit of $P_{i}$ is $\mathrm{MW}$, the voltage at the sending end is $U_{0}$, the voltage at the $i t h$ load's location of the line is $U_{i}$. The line impedance between the two adjoining loads is $R_{i-1, i}+j X_{i-1, i}$, the capacity of the PV integration injected into the $m t h$ load of the distribution network is $P_{P V m}$.

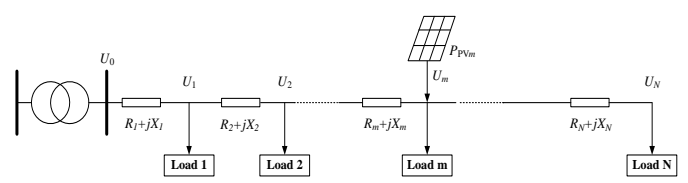

Fig1 A distribution grid with single point PV intergration 


\section{A typical distribution network with single PV integration}

As fig. 1 reports, the single PV integration is injected into the distribution network on the $m t h$ node. The PV is a unity power factor power source.

To the load node $N$ from $1^{\text {st }}$ to the $m t h$, the voltage difference between the $n t h$ node and the $(n-1) t h$ node andthe line loss between the two nodes as follow:

$$
\Delta U_{n}=U_{n-1}-U_{n}=\frac{\left(\sum_{i=k}^{N} P_{i}-P_{P V m}\right) R_{n}+\sum_{i=n}^{N} Q_{i} X_{n}}{U_{n-1}} ; \Delta S_{L n}=\frac{\left(\sum_{i=n}^{N} P_{i}-P_{P V m}\right)^{2}+\left(\sum_{i=n}^{N} Q_{i}\right)^{2}}{U_{n-1}^{2}}\left(R_{n}+j R X_{n}\right)
$$

Compared with the normal circumstances, the voltage of the feeder raises and the line loss changes after the injection of the PV integration, the amplitude of variation is related to the PV capacity, the loads and power line parameter. When the PV capacity is small, $\Delta U_{n}$ is a positive number, the voltage decreases along the direction of feeder, and the line loss reduces; With the increase of the PV capacity, $\Delta U_{n}$ turns to a negative number, that means the voltage of the $n t h$ node is higher than the previous one. If active power from the PV equals to the loads need. The line loss reaches the lowest level.After that, with the increase of active power from the PV, the line loss will increase again. The voltage of the $n t h$ load node can be evaluated as follows.

$$
\sum_{i=n}^{N} P_{i}=P_{P V m} ; \quad U_{n}=U_{0}-\sum_{k=1}^{n} \frac{\left(\sum_{i=k}^{N} P_{i}-P_{P V m}\right) R_{k}+\sum_{i=k}^{N} Q_{i} X_{k}}{U_{k-1}}
$$

To the load node $\mathrm{n}$ from the $m t h$ to the terminal one, the voltage difference between the $n t h$ node and the $(n-1) t h$ node is

$$
\Delta U_{n}=U_{n-1}-U_{n}=\frac{\sum_{i=n}^{N} P_{i} R_{n}+\sum_{i=n}^{N} Q_{i} X_{n}}{U_{n-1}}>0
$$

The line loss between the two nodes remains unchanged. So the voltage of the $n t h$ load node is:

$$
U_{n}=U_{0}-\sum_{k=1}^{m} \frac{\left(\sum_{i=k}^{N} P_{i}-P_{P V m}\right) R_{k}+\sum_{i=k}^{N} Q_{i} X_{k}}{U_{k-1}}-\sum_{k=m+1}^{n} \frac{\sum_{i=k}^{N} P_{i} R_{k}+\sum_{i=k}^{N} Q_{i} X_{k}}{U_{k-1}}
$$

Power loss of the entire feeder is:

$$
S_{L}=\sum_{n=1}^{n} \Delta S_{L n}=\sum_{n=1}^{m} \frac{\left(\sum_{i=n}^{N} P_{i}-P_{P V m}\right)^{2}+\left(\sum_{i=n}^{N} Q_{i}\right)^{2}}{U_{n-1}^{2}}\left(R_{n}+j R X_{n}\right)+\sum_{n=m+1}^{N} \frac{\left(\sum_{i=n}^{N} P_{i}\right)^{2}+\left(\sum_{i=n}^{N} Q_{i}\right)^{2}}{U_{n-1}^{2}}\left(R_{n}+j R X_{n}\right)
$$

From what has been discussed above, if the distribution network is connected with a single PV integration and voltage of the sending end keep unchanged, the variation of the voltage along the direction of feeder has four conditions. 1) consistently decreased; 2) decreased first then increased; 3) increased first then decreased: 4) consistently increased. In the last three cases, the highest voltage of the network appears on the PV access point.

$$
U_{t}=U_{0}-\sum_{k=1}^{m} \frac{\left(\sum_{i=k}^{N} P_{i}-P_{P V m}\right) R_{k}+\sum_{i=k}^{N} Q_{i} X_{k}}{U_{k-1}}
$$

The line loss from PV access point to the terminal remains unchanged. But in the meanwhile the line loss from the sending end to the access point decreases first then increases, because power flow decreases first then increases in the opposite direction with the increase of PV capacity.

It demands that voltage range of each load must meet the requirements of power quality indexes, and reducing the network loss to run distribution network economically is necessary. It is necessary to consider that step-down transformer is allowed to transmit power to the high voltage grid or not on the actual situation. So the maximum voltage of feeder after the injection of PV should less than the upper limit what is set by power quality indexes. 


$$
U_{m}=U_{0}-\sum_{k=1}^{m} \frac{\left(\sum_{i=k}^{N} P_{i}-P_{P V m}\right) R_{k}+\sum_{i=k}^{N} Q_{i} X_{k}}{U_{k-1}} \leq U_{\max }
$$

The upper limit is set based on power quality indexes, so the maximum capacity can be calculated under the constraint condition of static voltage stability.

\section{A typical distribution network with multi PV integration}

As fig.2 reports, it is assumed that multi PV integrations inject into the distribution network on each load node. If there is no PV integration on some nodes actually, the PV capacity can be equivalent to zero.

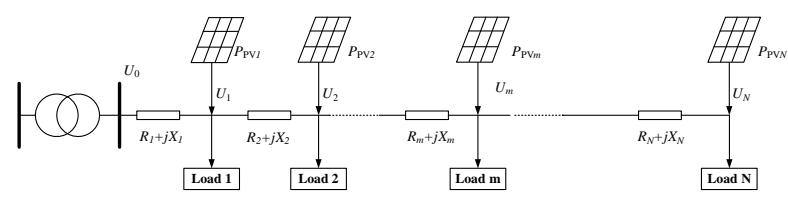

Fig2A distribution grid with multi point PV intergration

Then the voltage difference between the $n$th node and the $(n-1) t h$ node andt line loss between the two nodes is:

$$
\Delta U_{n}=U_{n-1}-U_{n}=\frac{\sum_{i=n}^{N}\left(P_{i}-P_{P V i}\right) R_{n}+\sum_{i=n}^{N} Q_{i} X_{n}}{U_{n-1}}, \Delta S_{\mathrm{L} n}=\frac{\left(\sum_{i=n}^{N}\left(P_{i}-P_{P V i}\right)\right)^{2}+\left(\sum_{i=n}^{N} Q_{i}\right)^{2}}{U_{n-1}^{2}}\left(R_{n}+j R X_{n}\right) \text { (8) }
$$

So if $\Delta U_{n}$ is a positive number, the voltage decreases along the direction of feeder. If $\Delta U_{n}$ turns to a negative number, that means the voltage of the $n t h$ node is higher than the previous node, the voltage increases along the direction of feeder. The highest voltage of the network is related to the PV capacity, the loads and power line parameter andThe voltage of the $n t h$ node as follows:

$$
U_{n}=U_{0}-\sum_{k=1}^{n} \frac{\left(\sum_{i=k}^{N}\left(P_{i}-P_{P V i}\right)\right) R_{k}+\sum_{i=k}^{N} Q_{i} X_{k}}{U_{k-1}} \quad ; \sum_{i=k}^{N} P_{i}=\sum_{i=k}^{N} P_{P V i}
$$

The line loss is related to the sum of the PV capacity from the access point node to the terminal one. The network loss decreases with the sum of PV capacity increases from zero. When the sum of PV capacity is equal to load's active power, the line loss reaches the minimum.

Then, the line loss increases as the sum of PV capacity raises more.

$$
S_{L}=\sum_{n=1}^{n} \Delta S_{L n}=\sum_{n=1}^{N} \frac{\left(\sum_{i=n}^{N} P_{i}-P_{P V i}\right)^{2}+\left(\sum_{i=n}^{N} Q_{i}\right)^{2}}{U_{n-1}^{2}}\left(R_{n}+j R X_{n}\right)
$$

\section{Model and Simulation}

The distribution network in fig. 1 is used as an example to find out the impact of large-scale PV integration on voltage and line loss of distribution network. The voltage of the sending end is $10.5 \mathrm{KV}$, transmission line voltage is $10 \mathrm{KV}$. According to power quality indexes, the voltage on each load node can't exceed 10.7KV. 10 nodes are evenly distributed on the feeder. The interval is 100 meter between two adjacent spaced-load, and load power factor is 0.98 . All of the loads are connected by aerial line using aluminiumwire, the line impedance is $1.285+\mathrm{j} 0.395 \Omega / \mathrm{km}$ in a unit of length.

\section{Simulation of a typical distribution network with single PV integration}

Assuming each load is $15 \mathrm{kVA}$, the fifth load node was connected with different PV. Fig.3 shows the practical distribution of voltage on the feeder.Assuming the size of load doesn't change, a PV whose capacity is 3MW was connected with different node. Fig.4 shows the practical distribution of voltage on the feeder. 


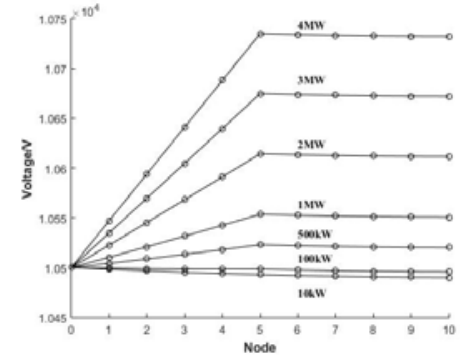

Fig3 Feedervoltage with different PV at

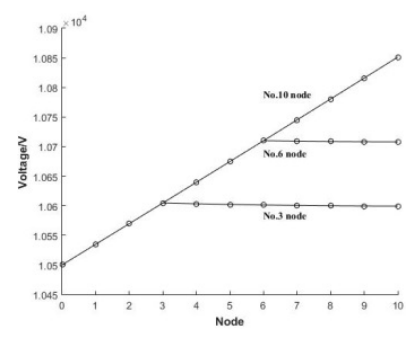

Fig4 Feeder voltage with 3MW PV at different point

The load capacity has influence on the distribution of voltage. Assuming each load changes from $15 \mathrm{kVA}$ to $500 \mathrm{kVA}$, and load power factor is 0.98 . Fig. 6 shows the practical distribution of voltage on the feeder when the fifth node is connected with a PV whose capacity is 4MW.

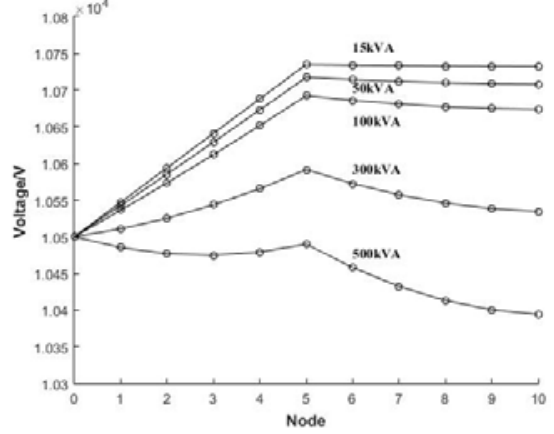

Fig5 Feeder voltage variation with loads

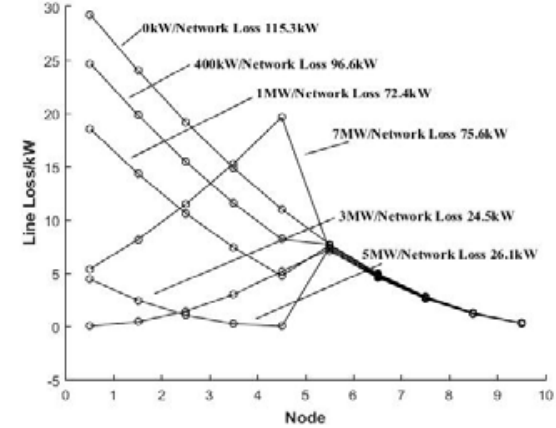

Fig6 Network loss with different load

Three conclusions are as fellows.

With the increase of PV capacity, the voltage on PV access point and the surroundings increase. And the distribution of voltage along the direction of feeder has four forms: 1) consistently decreased; 2) decreased first then increased; 3) increased first then decreased; 4) consistently increased. According to power quality indexes, the voltage can't exceed $10.7 \mathrm{kV}$, so the maximum capacity of PV injected into the fifth node is about 3.5MW (fig.3).

Assuming each load is 500kVA, fig.6 shows the feeder loss when the fifth node is connected with different PV capacity. As can be seen in the figure, when the capacity is small, the network loss decrease with the capacity increased. When the capacity is equal to the sum of active power from the total load, the feeder loss from the seeding end to the access point turn decreased to increased, but the feeder loss from the access point to the terminal remains the same.

Assuming each load is still 500kVA, fig.7 shows the feeder loss when a PV whose capacity is 3MW access to different load node. As can be seen in the figure, when the access point is approaching to the middle part, it has more obvious effects reducing the network loss.

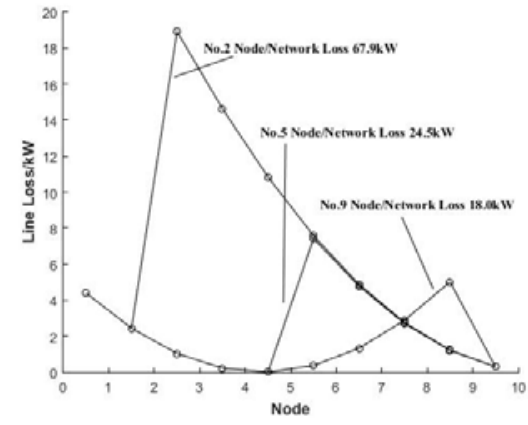

Fig7 Network loss with 3MW PV at different load

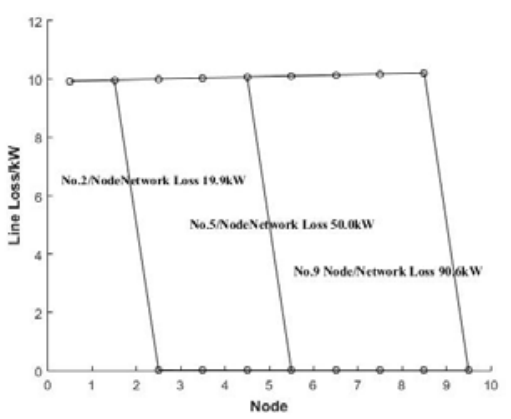

Fig8 Network loss with different loads

Assuming each load is 15kVA, fig.8 shows the feeder loss when a PV whose capacity is 3MW access to different load node. As can be seen in the figure, the loss is small when the access point is 
near to the sending end. Compared to the figure above, if the PV capacity is small compared with each load's size, it has more obvious effects reducing the network loss when the access point is approaching to the terminal. And the best intervention point moves forward to the sending end with the PV capacity increases.

\section{Simulation of a typical distribution network with multi PV integration}

Fig.9 shows the simulation when each load is 100kVA and each node is injected with PV having the same capacity. The voltage of each node increases with the increase of PV capacity, especially the terminal voltage raises greatly.

If the PV capacity injected to each node gets to $400 \mathrm{~kW}$, the terminal voltage gets to the limit value $10.7 \mathrm{kV}$. So the maximum PV capacity allowed to be injected to the network is $4 \mathrm{MW}$ in this case.

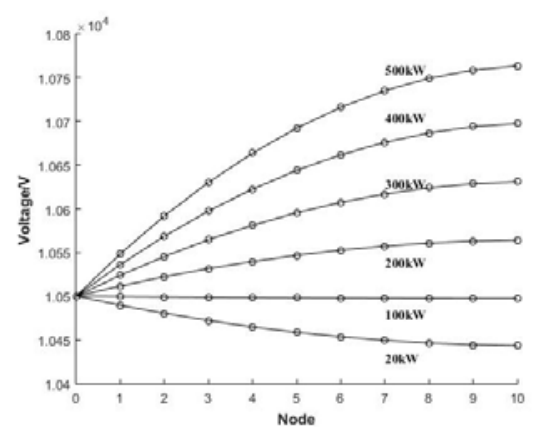

Fig9 Feeder voltage with PV connected at different nodes

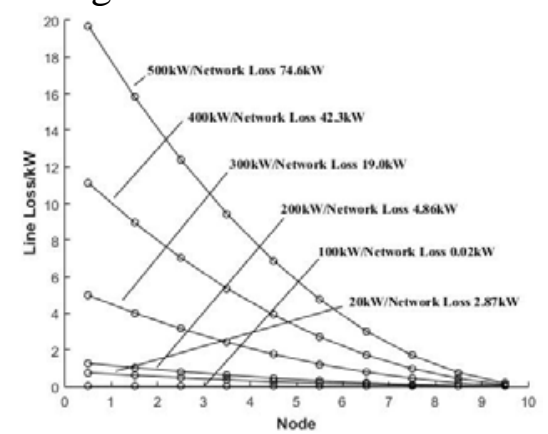

Fig10 Network loss with PV connected at different nodes

Fig.10 shows the feeder loss in the same case. It's easy to see that feeder loss is concerned with the difference between the load and the PV capacity, when the sum of PV capacity is near to the load, the feeder loss reaches the minimum.

\section{Conclusion}

In this paper, by means of analytical expressions and simulation, the variation of voltage amplitude and feeder loss when distribution network is connected with large-scale PV is investigated. There are some main important conclusions.

(1) When the PV capacity injected to the distribution network is big, the voltage of access point will rise even passed the limit. The feeder loss increases first then decreases with the increase of PV capacity. When distribution network is connected with multi PV integration, the variation of voltage is concerned with the difference between the load and the PV capacity, when the sum of PV capacity is close to the load, the feeder loss reaches the minimum.

(2) The variation of the voltage along the direction of feeder has four conditions. 1) consistently decreased; 2) decreased first then increased; 3) increased first then decreased; 4) consistently increased. In the last three cases, the highest voltage in the network appears on the access point, the maximum PV capacity can be determined based on this. The highest voltage in the distribution 
network with multi PV integration is concerned with each load and PV capacity. The maximum capacity is determined based on voltage not passing the limit.

\section{Acknowledgment}

Grateful acknowledgement is made to the support of the 5210EF140002 item from State Grid Jiangsu Electric Power Company.

\section{References}

[1] Cavlovic, M., "Challenges of optimizing the integration of distributed generation into the distribution network," in Energy Market (EEM), 2011 8th International Conference on the European , vol., no., pp.419-426, 25-27 May 2011

[2] Liu, Y.; Bebic, J.; Kroposki, B.; de Bedout, J.; Ren, W., "Distribution System Voltage Performance Analysis for High-Penetration PV," in Energy 2030 Conference, 2008. ENERGY 2008. IEEE , vol., no., pp.1-8, 17-18 Nov. 2008

[3] Shahnia, F.; Ghosh, A., "Decentralized voltage support in a Low Voltage feeder by droop based voltage controlled PVs," in Power Engineering Conference (AUPEC), 2013 Australasian Universities , vol., no., pp.1-6, Sept. 29 2013-Oct. 32013

[4] Masoum, A.S.; Moses, P.S.; Masoum, M.A.S.; Abu-Siada, A., "Impact of rooftop PV generation on distribution transformer and voltage profile of residential and commercial networks," in Innovative Smart Grid Technologies (ISGT), 2012 IEEE PES , vol., no., pp.1-7, 16-20 Jan. 2012

[5] Madureira, A. G., \& Lopes, J. P. (2009). Coordinated voltage support in distribution networks with distributed generation and microgrids. IET Renewable Power Generation, 3(4), 439-454.

[6] Madureira, A.G.; Pecas Lopes, J.A., "Coordinated voltage support in distribution networks with distributed generation and microgrids," in Renewable Power Generation, IET , vol.3, no.4, pp.439-454, December 2009 